\title{
Villon et ses lecteurs. Actes du colloque international des 13-14 décembre 2000 organisé à la Bibliothèque historique de la Ville de Paris, Textes édités par Jean Dufournet, Michael Freeman et Jean Derens
}

\section{Maria Colombo Timelli}

\section{OpenEdition}

\section{Journals}

Édition électronique

URL : http://journals.openedition.org/studifrancesi/27272

DOI : 10.4000/studifrancesi.27272

ISSN : 2421-5856

Éditeur

Rosenberg \& Sellier

Édition imprimée

Date de publication : 31 décembre 2006

Pagination : $581-583$

ISSN : 0039-2944

Référence électronique

Maria Colombo Timelli, «Villon et ses lecteurs. Actes du colloque international des 13-14 décembre 2000 organisé à la Bibliothèque historique de la Ville de Paris, Textes édités par Jean Dufournet, Michael Freeman et Jean Derens », Studi Francesi [En ligne], 150 (L | III) | 2006, mis en ligne le 30 novembre 2015, consulté le 08 novembre 2020. URL : http://journals.openedition.org/studifrancesi/ 27272 ; DOI : https://doi.org/10.4000/studifrancesi.27272

Ce document a été généré automatiquement le 8 novembre 2020

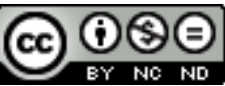

Studi Francesi è distribuita con Licenza Creative Commons Attribuzione - Non commerciale - Non opere derivate 4.0 Internazionale. 


\title{
Villon et ses lecteurs. Actes du colloque international des 13-14 décembre 2000 organisé à la Bibliothèque historique de la Ville de Paris, Textes édités par Jean Dufournet, Michael Freeman et Jean Derens
}

\author{
Maria Colombo Timelli
}

\section{RÉFÉRENCE}

Villon et ses lecteurs. Actes du colloque international des 13-14 décembre 2000 organisé à la Bibliothèque historique de la Ville de Paris, Textes édités par Jean DUFOURNET, Michael FREEMAN et Jean DERENS, Paris, Honoré Champion, 2005 («Colloques, congrès et conférences sur le Moyen Âge», 5), pp. 337.

1 En introduisant les travaux de ce Colloque, dont les Actes paraissent avec quelque retard, Jean DUFOURNET rappelle quelques ouvrages significatifs publiés entre 1995 et 2002 diversement inspirés par F.V. (Présentation, pp. 11-15) Tous les domaines sont concernés: de la critique universitaire à la littérature (romans et scénarios), à la production dramatique, pour ne rien dire des disques et des expositions. Comme le dit J.D., "Villon est donc partout» (p. 15). Ce volume se veut un parcours consacré à quelques-uns des innombrables lecteurs du poète et des lectures qu'il a suscitées du $\mathrm{XV}^{\mathrm{e}}$ siècle à nos jours. 
2 Ce panoramique s'ouvre sur l'édition de V. fournie par Clément Marot en 1532. Jacqueline CERQUIGLINI-TOULET (Marot et V., pp. 19-31) souligne les éléments de proximité (thèmes poétiques et sensibilité à l'égard de l'évolution de la langue) et de distance (origine, formation, esthétique) entre les deux poètes. Marot éditeur est aussi philologue dans son respect du texte, et critique littéraire par son souci de l'histoire et de l'attribution des poèmes: c'est lui, selon les mots de J.C.-T. qui «offre à V. ses lettres de noblesse et sa place dans l'histoire de la littérature» (p. 31).

3 Jean Devaux (La Male Fortune de V. aux Grands Rhétoriqueurs, pp. 35-51) étudie la figure de (Male) Fortune chez Villon (Ballade de Fortune et Bergeronnecte après le huitain CLXVI du Testament) et chez les Grands Rhétoriqueurs des années 1464-1490. Il met en relief traits communs (la nature malfaisante de Fortune et, dans une certaine mesure, sa relation avec les desseins de Dieu) et surtout les différences d'interprétation entre un Villon angoissé devant les inconséquences et la «desraison» de Fortune et des poètes moralisants, souvent marqués par un solide conformisme, chez lesquels dominent le motif de la mutabilité de Fortune, mais aussi la possibilité pour l'homme de lui faire face grâce à «franc arbitre».

4 Jelle KoOpmans ('Mort, j'appelle ta rigueur', ou la première Renaissance française à l'école de F.V., pp. 55-67) rappelle que le rondeau 'Mort, j'appelle...' a circulé de manière isolée comme chanson autonome, accompagnée ou non de notation musicale, avant ou après l'intégration au Testament. Les manuscrits concernés (le Chansonnier Nivelle la Chaussée, le ms. 517 de la B.M. de Dijon, le Chansonnier Laborde, le ms. La Haye, B.R. 129.G.20, le Chansonnier Rohan) apportent la preuve d'une transmission du texte appuyée sur l'écoute bien plus que sur la lecture.

5 Avec Robert D. PЕскнAM on aborde le siècle des Lumières et sa réception de V. (A' la recherche d'un V. perdu. Pour une histoire de sa réception au XVIIIe siècle, pp. 71-88: vaste bibliographie aux pp. 80-88). En se fondant sur des éléments codicologiques incontestables et sur la complémentarité du contenu, R.D.P. attribue à Nicolas LengletDufresnoy la paternité du ms. Londres B.L. 241.f.17 (édition des «Poésies de F.V.») et du ms. Arsenal 2948 («Notes sur les Poésies de F.V.»). Deux questions demeurent ouvertes: pourquoi Lenglet a abandonné son projet d'édition, et comment / pour quelles raisons les deux volumes ont été séparés.

6 Emmanuèle BAUMgaRTNER étudie Théophile Gautier lecteur de V. (pp. 91-102). Dans un article publié en 1834, Gautier présente l'intégralité de l'œuvre de V. dans le but implicite de réhabiliter, avec le poète $\mathrm{du} \mathrm{XV}^{\mathrm{e}}$ siècle, les écrivains modernes injustement méprisés. Parmi les mérites de V., Gautier indique le refus des stéréotypes et le choix d'une poésie «neuve, forte et naïve»; il lit donc l'ensemble de l'œuvre comme le reflet authentique d'une vie bien réelle. Cependant, tout en tombant dans le piège de l'illusion biographique, Gautier est peut-être le seul de son temps à reconnaître certains traits de l'art de $\mathrm{V}$.

7 Baudelaire n'a consacré à V. qu'une rapide allusion dans son essai Quelques caricaturistes français. Cependant, selon Michael EDWARDS (V. et Baudelaire, pp. 106-116), les deux poètes se ressemblent beaucoup par leur 'inconduite' ('delinquencies' en anglais) et, malgré de profondes différences, par la manière de se présenter au lecteur dans leurs poèmes.

8 Le riche panoramique dressé par Bertrand DEGOTT des poètes auteurs de ballades à partir de 1850, Théodore de Banville au premier chef ('Au nom du frère'. V. dans la ballade 
française après 1850, pp. 119-136), montre à l'évidence combien et comment F.V. est lu, traduit, renouvelé voire parodié. Tant dans la forme que dans les sujets et même dans le choix des rimes, il constitue un intertexte essentiel et permet de réaffirmer une tradition lyrique bien française.

Michael fREEMAN ('L'étrange cas du docteur Stevenson et de Monsieur V.', pp. 139-154). Bon connaisseur de la langue et de la littérature françaises, francophile déclaré, R.L. Stevenson est l'auteur d'une nouvelle et d'un essai sur F.V., tous les deux publiés en 1877. Il s'agit d'œuvres de jeunesse (Stevenson était né en 1750), qui montrent la fascination exercée par le poète français du XV siècle sur le jeune calviniste du XVIII ${ }^{e}$. Dans la nouvelle, est brossé un portrait imaginaire du poète-voleur, alors que l'essai, écrit sous l'influence du livre d'A. Longnon paru la même année, en fait un homme de génie, un des plus grands poètes de la France, dont l'influence se fait toujours sentir: aux yeux de Stevenson, $V$. incarne dans une certaine mesure le génie même de la nation française.

10 Jean DÉRENS (Les tribulations d'Auguste Longnon, découvreur de V., pp. 157-163) rappelle les recherches menées par A. Longnon pendant une vingtaine d'années et son édition de Villon (1892), en citant parallèlement les comptes rendus et 'chroniques' de Gaston Paris publiés dans la Romania.

11 Jean DUFOURNET et Sylvie NÈVE (Marcel Schwob et V., pp. 167-204) proposent une longue étude en trois volets sur la fréquentation assidue de F.V. par Marcel Schwob, écrivain divers s'il en est: (1) recherche d'un V. 'total' (vie et œuvre), (2) études sur l'argot, ayant déterminé le regard de Schwob sur la poésie et la personnalité villoniennes, (3) reconstitution du milieu historique, géographique et social du poète. En annexe, le Catalogue 'villonien' de la bibliothèque de M. Schwob (n.os 715-743) et les Extraits du Procès des Coquillars tirés du tome VI de ses Euvres complètes.

12 Michel DECAUdin (Apollinaire et V., pp. 207-221). Apolinnaire a aussi entretenu une relation précoce et durable avec $V$., dont témoignent l'œuvre critique et surtout l'œuvre poétique: les convergences thématiques et les affinités existentielles ont été maintes fois soulignées. En annexe, un document inédit: la copie fragmentaire, faite par Apollinaire en 1899, du Testament à partir de l'édition de 1532 conservée à Lyon.

De V. à Cendrars, c'est une véritable 'filiation' qui se manifeste selon Luisa MONTROSSET (Cendrars ascendant V.: herméneutique d'une filiation zodiacale, pp. 225-238). Par l'affinité de l'inspiration et du code poétique, nombreux sont les endroits de l'œuvre de Cendrars marqués par l'influence d'un de ses écrivains préférés.

Pierre CAizergues (Cocteau et V., pp. 241-248). Si les occurrences du nom de V. dans l'œuvre de Cocteau ne sont pas nombreuses, celui-ci est néanmoins l'auteur d'un poème À la Villon, édité ici pour la première fois (pp. 247-248).

Jane H.M. TAYLOR (Le défi de la modernité: F.V. et Basil Bunting, pp. 251-266). Basil Bunting est l'auteur anglais d'un poème, Villon (1925), texte hybride reproduit ici (pp. 262-266), qui exige des lecteurs capables de reconnaître les nombreux emprunts - sous la forme d'allusions et de citations - au Testament. Sous l'influence d'Ezra Pound, Bunting établit une profonde relation entre lui-même et un V. romantique, emblème de la liberté de l'homme et du poète.

16 François DE LA BRETÈQUE (V. au cinéma, pp. 269-292) a recensé huit films où F.V. est mis en scène (filmographie aux pp. 290-292); il distingue d'abord un V. 'français' et un V. 'américain', mais conclut à une perméabilité des deux modèles. 
17 Giuseppe BRUNELLI (Lecteurs italiens de V. (1906-2000), pp. 295-302) offre un panoramique de la 'réception' de V. en Italie au $\mathrm{XX}^{\mathrm{e}}$ siècle: traductions, études scientifiques et universitaires, mais aussi romans et pièces de théâtre inspirées à la vie du poète.

V. est enfin présent dans la vie culturelle polonaise après 1945: Pierre DEMAROLLE (V. et ses lecteurs polonais, pp. 305-318) donne un aperçu des éditions et traductions de son œuvre, mais encore des pièces théâtrales, émissions radio et télé, qui ont fait place à V., dont des ballades ont aussi été mises en musique. Quatorze poèmes du $\mathrm{XX}^{\mathrm{e}}$ siècle, présentés ici en traduction française, montrent le rayonnement de la poésie de $\mathrm{V}$. auprès du public privilégié formé par les poètes polonais.

Trouvent aussi place, entre un essai et l'autre, des ballades modernes et contemporaines diversement inspirés par le poète $\mathrm{du} \mathrm{XV}^{\mathrm{e}}$ siècle; les auteurs en sont: Théodore de Banville (1892), Alcanter de Brahm (1937), Paul Marissiaux (1930), Catulle Mendès (1900), Emile Bergerat (1910), Jean-Marc Bernard (1923), Cyrille Dubus (1966), Alphonse Piché (1946), Fagus (1926), Henri Galoy (1897), Raoul Ponchon (1886), Renée Humbert-Gley (1932), Ernest Jaubert (1908), Ferdinand Lovio (1923), Torrente (1911), Victor Margueritte (1885); auxquels s'ajoutent deux des participants au Colloque: Michael Edwards et Bertrand Degott (auteur de la Ballade du Colloque, datée 14 décembre 2002, qui clôt le volume).

Index des noms de personnes et de personnages fictifs aux pp. 323-333. 